\title{
An early-stage classification of Lung Nodules by an Android based application using Deep Convolution Neural Network with Cost-sensitive loss function and Progressive scaling approach
}

\author{
Mrudang D. Pandya ${ }^{1}$, Dr. Sunil Jardosh ${ }^{2}$, Dr. Amit R. Thakkar ${ }^{3}$ \\ ${ }^{1}$ Assistant Professor, Department of Information Technology, \\ CHARUSAT Deemed to be University, Anand, Gujarat, India, mrudangpandya.it@ charusat.ac.in \\ ${ }^{2}$ Senior Project Manager, \\ Progress Software, Hyderabad, India, sunil.jardosh@gmail.com \\ ${ }^{3}$ Associate Professor, Department of Information Technology, \\ CHARUSAT Deemed to be University, Anand, Gujarat, India, amitthakkar.it@charusat.ac.in
}

\begin{abstract}
Minimization of the death proportions of lung cancer there is a requirement of a computer-aided diagnosis (CAD) system for early-stage detection and classification of lung nodules. This paper has proposed two novel approaches based on deep learning techniques improvement of the classification accuracy of lung nodules in computed tomography (CT) scans. Our first approach uses Two-dimensional Convolution Neural Network architecture for automatic feature extraction and classification of lung candidates as cancer nodules or noncancerous nodules. In the second approach, we have used the progressive scaling technique in which we have started with small size images( $\mathrm{n}$ $n$ ) and train the model than we gradually increase the size of image( $I n$ the ratio of $2 n \times 2 n$ ) and train again this approach give promising result to increase classifier result. We have measured our approaches on the Lung Image Database Consortium image collection (LIDC/IDRI) dataset expected by the LUNA16 challenge. After experimenting with models we have created an android mobile application using the TensorFlowlite framework which is taking lung CT scan as an input and produce benign or malignant probability in the given scan. Experimental results proved that our deep learning architecture with a combination of cost-sensitive loss function and augmentation of minority class has produced an accuracy of $96.9 \%$, the sensitivity of $96.2 \%$, and specificity of $97.2 \%$. In the second approach of progressive resizing, we have graduated with an increase in the testing accuracy.
\end{abstract}

Key words: Computer-aided diagnosis, Cost-sensitive function, Tensorflowlite, Alexnet, 2DCNN, LUNA16, Progressive resizes, Sensitivity.

\section{INTRODUCTION}

As per the survey of the Indian Council of Medical Research (ICMR), there were 1.45 million new cancer cases detected and as per the prediction of ICMR, this will increase up to 1.73 million in 2020. Among these lung cancers: an estimated 0.114 million (83,000 in males and 31,000 in females) new cases during 2016 and that will increase to 0.14 million in 2020[3]. Another survey has done by the American Cancer Society (ACS) in 2012[1]. They have found a total of 1800000 cases of lung cancer all over the world. The below Table I indicates the number of lung cancer cases percentage-wise in different coun- tries. Lung cancer is the second most common cancer in American men and women. It's also the leading cause of cancerrelated deaths for both American men and women. One in every four cancer-related deaths is from lung cancer. The main cause of lung cancer is considered to be cigarette smoking. There is 23 times in men \& 13 times in women more chance of developing lung cancer, who smokes. About $14 \%$ of new cancer cases in the United States are lung cancer cases. That equals about 234,030 new cases of lung cancer each year. There are two main types of lung cancer: 1. Non-small cell lung cancer (NSCLC) 2. Small cell lung cancer(SCLC). Roughly $85 \%$ of people diagnosed with lung cancer each year have NSCLC and rest have SCLC. The average age of a lung cancer diagnosis is 70 , with the majority of diagnoses in adults over the age of 65 . A very small number of lung cancer diagnoses are made in adults under age 45 .

Table 1: Lung cancer survey by ACS [1]

\begin{tabular}{|c|c|}
\hline Country & Lung cancer cases (\%) \\
\hline China & 35.8 \\
\hline Europe & 22.5 \\
\hline Middle East \& North Africa & 2.9 \\
\hline India & 3.9 \\
\hline Northern America & 13.1 \\
\hline Sub-Saharan Africa & 0.9 \\
\hline Other East \& Central Asia & 15.6 \\
\hline Latin America \& the Caribbean & 4.5 \\
\hline Oceania & 0.8 \\
\hline
\end{tabular}

Mainly there are four levels of Lung Cancer stages. If we talk about the survival rate of both the types concerning levels, Table $2 \&$ has given five-years survival rate percent.

Table 2: NSCLC survival rate

\begin{tabular}{|c|c|}
\hline Stage & Five-year survival rate \\
\hline 1A & 92 percent \\
\hline 1B & 68 percent \\
\hline 2A & 60 percent \\
\hline 2B & 53 percent \\
\hline 3A & 36 percent \\
\hline 3B & 26 percent \\
\hline 4, or metastatic & 10 percent, or $<1 \%$ \\
\hline
\end{tabular}


Table3: SCLC survival rate

\begin{tabular}{|c|c|}
\hline Stage & Five-year survival rate \\
\hline 1 & 31 percent \\
\hline 2 & 19 percent \\
\hline 3 & 8 percent \\
\hline 4, or metastatic & 2 percent \\
\hline
\end{tabular}

So, early cancer detection and survival can be the biggest issue in the medical domain. Lung cancer is that type of cancer that is largely detected not only in India but all over the world. After diagnosed with lung cancer in the US, the patient cannot survive more than 5 years. The survival rate is only $17 \%$ and this is even lesser in developing countries [2]. There were different data modalities available for the detection of lung cancer in the medical image domain (ex. MRI, CT, PET) but we have selected CT scan images in the form of DICOM, RAW, etc. formats because These high-tech scans produce 3D images. These scans can not only identify tumors earlier but also spot them when the tumors are smaller and more treatable by surgery. According to Doi[3], 30\% of lung nodules may be missed by the radiologists due to overlaps between them and other normal anatomic structures. For the automation and early detection of lung cancer, we have tried to detect the same with the use of some state-of-art techniques[21, 22] of Deep Learning especially 2D and 3D Convolution Neural Networks (CNNs) and tried to apply the concept of transfer learning for the early-stage detection of lung cancer. The challenges we have faced like the development of such an algorithm that accurately determines when lesions in the lungs are cancerous. Also, the reduction of the false-negative rate was a challenging task. The overall goal of our research is to develop such algorithm or system by which we can predict early-stage cancer and increase the survival rate of patients. Radiologists can get more time with the patients. As per the Torre et al. [4] research, nodules may be tiny $(<10 \mathrm{~mm})$ or big $(>30 \mathrm{~mm})$. To find out tiny nodules it is like we have to find out a $10 \mathrm{~mm}$ nodule in the search space of $400 * 400 * 200$. Additionally, CT scans have lots of noise values in terms of air, bone, tissues, water, blood, etc. So basic lung cancer classification process we have to follow is described in fig. 1.

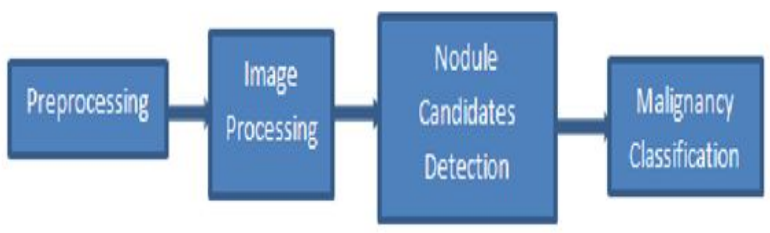

Figure 1: Lung Cancer classification pipeline

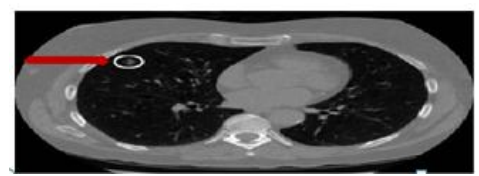

Figure 2: Lung Nodule example

Our objective is to enhance the precision of CADe systems by exploiting the advantage of deep learning [9] and to provide more mobility transform that system into a mobile application using the TensorFlowlite framework. In detail, we propose a new 2D deep convolutional neural network architecture with the cost-sensitive loss function and augmentation techniques for boosting classification accuracy and provide a more sensitive model towards lung cancer detection. Our $2^{\text {nd }}$ approach of progressive resizing also has produced good classification results. To provide better feasibility to the radiologists we have created a TensorFlowlite framework based android mobile application.

\section{RELATED WORK}

For a deep understanding of image-related data with the use of the convolution process convolution neural network has provided advancement in artificial neural networks[23]. This network is inspired by the human biological visual structure so it works very effectively and efficiently for the problems of image recognition[22]. It also works irrespective of image size and scale. This architecture has provided a "neighbors" approach instead of a fully connected network that was there in the fully connected artificial neural network architecture. Due to this "neighbors" approach, we can reduce the number of connections of neurons or the number of weight much lesser than the fully connected network [10]. By this, we can reduce the problem of over fitting which is very common in fully connected networks. It will also take less time for training concerning fully connected architecture. CNN has many variations in terms of the number of layers. In below table 4 we have listed some of the variations of CNN architectures. These variations are invented to solve the ImageNet Large Scale Visual Recognition Challenge (ILSVRC) annual contest.

Table 4: ILSVRC competitioncnn architecture variances [18]

\begin{tabular}{|c|c|c|c|c|c|}
\hline Year & $\begin{array}{l}\text { CNN } \\
\text { Varia- } \\
\text { tions }\end{array}$ & $\begin{array}{l}\text { Devel- } \\
\text { oped by }\end{array}$ & $\begin{array}{l}\text { Ran } \\
\mathbf{k}\end{array}$ & $\begin{array}{l}\text { Top-5 } \\
\text { error } \\
\text { rate }\end{array}$ & $\begin{array}{l}\text { No. of } \\
\text { para- } \\
\text { meters }\end{array}$ \\
\hline 1998 & $\begin{array}{l}\text { Le- } \\
\text { Net(8) }\end{array}$ & Yannlecun & & & $\begin{array}{l}60 \\
\text { Thou- } \\
\text { sand }\end{array}$ \\
\hline 2012 & $\begin{array}{l}\text { Alex- } \\
\text { Net(7) }\end{array}$ & $\begin{array}{l}\text { Alex Kriz- } \\
\text { Kriz- } \\
\text { hersky,Ge } \\
\text { offreyHin- } \\
\text { ton,llyasut } \\
\text { skever }\end{array}$ & $1^{\mathrm{st}}$ & $\begin{array}{l}15.39 \\
\%\end{array}$ & $\begin{array}{l}60 \\
\text { million }\end{array}$ \\
\hline 2013 & ZFNet() & $\begin{array}{l}\text { Matthew } \\
\text { Zeiler and } \\
\text { Rob Fer- } \\
\text { gus }\end{array}$ & $1^{\mathrm{st}}$ & $\begin{array}{l}14.89 \\
\%\end{array}$ & - \\
\hline 2014 & $\begin{array}{l}\text { Google- } \\
\text { le- } \\
\text { Net(19) }\end{array}$ & Google & $1^{\mathrm{st}}$ & $6.67 \%$ & $\begin{array}{l}4 \text { mil- } \\
\text { lion }\end{array}$ \\
\hline 2014 & $\begin{array}{l}\text { VGGNe } \\
\mathrm{t}(16)\end{array}$ & $\begin{array}{l}\text { Simo- } \\
\text { nyan,zisse } \\
\text { rman }\end{array}$ & $2^{\text {nd }}$ & $7.39 \%$ & $\begin{array}{l}138 \\
\text { million }\end{array}$ \\
\hline 2015 & $\begin{array}{l}\text { Res- } \\
\text { Net(15) }\end{array}$ & $\begin{array}{l}\text { Kaiming } \\
\mathrm{He}\end{array}$ & $1^{\mathrm{st}}$ & $3.69 \%$ & \\
\hline
\end{tabular}

Manipulating the benefits of deep learning [7, 8], several methods are presented to increase the classification accuracy of lung nodule candidates in CT scans [5]. Here we have given information about the researcher and there results for lung nodule detection and classification in terms of accuracy, sensitivity, and specificity.

Giang et al. [6, 11] proposed 15-layers 2D-CNN architecture with the CNN blocks approach. To increase classification accuracy they have introduced focal loss function instead of $\mathrm{BCE}$ 
function. The proposed method can gain up to $97.2 \%$ accuracy and $96.0 \%$ sensitivity and 97.3 specificity of lung nodule classification.

$\mathrm{Li}$ and Cao et al. [12] projected a 2D-DCNN to classify nodule candidates as a nodule or non-nodule. The suggested system was trained and validated on 62,492 regions-of-interest (ROI) image patches mined from 1,010 CT scans of the LIDC/IDRI dataset. The proposed method has produced $86.4 \%$ accuracy and $89.0 \%$ sensitivity of lung nodule classification.

Kuruvilla [13] proposed a CAD system for the classification of lung nodule cancer. The tests confirmed that the method obtains an accuracy of $93.3 \%$, a sensitivity of $91.4 \%$, and specificity of $100 \%$. For determining non-nodule this method showed very promising results.

W-J Choi and T-S Choi [14] presented a lung nodule detection system using hierarchical block classification. The proposed method shows a very good accuracy of $97.6 \%$, a sensitivity of $95.2 \%$, and a specificity of $96.2 \%$ for lung nodule detection.

Setio et al. [15] proposed multiview CNN (ConvNets) to classify lung nodule candidates. The method was trained on LIDC/IDRI dataset by the LUNA16 challenge. The tests show that the method able to achieve a sensitivity of $90.1 \%$. Torres et al. [16] used a feed-forward neural network (FFNN) as a nodule candidate classifier. The training and testing of the application has done on the LIDC/IDRI dataset. Results showed sensitivity up to $89.1 \%$. [17] S. G. Armato et al. have used 1,186 lung nodules from LIDC-IDRI chest CT images and delivered these nodules as positive candidates for researchers. The results of the challenge show that the best distinct recognition system can obtain up to $92.9 \%$ sensitivity of lung nodule detection.

\section{RESOURCES AND APPROACHES}

\subsection{Data}

In our research, we have used the LUNA16 challenge dataset which was extracted from the LIDC/IDRI dataset [19]. This dataset matched with our objective of lung nodule classification. So we have used that dataset for our research. The dataset includes 888 thoracic CT scans with annotation .csv file and candidates.csv file. In this annotation.csv file 1186 patients ID with $\mathrm{X}, \mathrm{Y}, \mathrm{Z}$ coordinates and cancerous nodule diameter in $\mathrm{mm}$ suggested by 4 different radiologists. The second file available with the dataset is candidates file in which includes 551066 nodules among these probable nodules only 1351 nodules marked as cancerous nodules. So there was lots of imbalance in data.

In our research, we use 1351 positive candidates and 5549715 negative candidates to detect lung nodules. The numbers of positive and negative nodules candidates are extremely imbalanced for training and testing models. To handle this kind of imbalance we have used data augmentation techniques that we describe in the next session.

\subsection{Data Augmentation:}

To handle data imbalance we have decided to under-sample the majority class and augment the minority class [11]. There are 1069 samples available for positive class (we have removed some samples which are very near to lung boundaries). For training the model we have augmented positive samples two times more so in total 3207 samples and under the majority classes such that we can have 1:3 distributions. So finally we have 12828 total samples among them non-nodule class samples 9621 and nodule class samples 3207. For augmentation of minority class we have performed below operations:
1. Rotation : $180,90,45$ degree random rotation

2. Horizontal flip

3. Vertical flip

4. Horizontal and vertical shift

We elect not to apply non-deviating alterations, like stretching or skewing, zooming, blurring, for the significance of nodule's shape for the detection process.

\subsection{Structure of CNN Architecture}

We have created two different architectures for our training process. The design of the architectures is given in Fig. 3 (Model-1[M-1]) and Fig. 4(Model-2[M-2]). Model-1 consists of 7 2D-convolutions (Conv2D) layers each layer followed by Batch- Normalization function and ReLU activation function. $1^{\text {st }}, 4^{\text {th, }}$ and $5^{\text {th }}$ Conv2D layers have 64 numbers of filters, $2^{\text {nd }}$ and $6^{\text {th }}$ Conv2D layers have 128 numbers of filters and $3^{\text {rd }}$ and $7^{\text {th }}$ Conv2D layers have 512 numbers of filters. After Conv2D layers next layer is flatten layer after that there are 3 fully connected (FC) layers. $1^{\text {st }} \mathrm{FC}$ having 512 nodes, $2^{\text {nd }} \mathrm{FC}$ having 128 nodes and the end we have 2 nodes and sigmoid activation function that will classify and give probabilities of input being in a nodule or non-nodule class. Model-2 having Conv2D blocks instead of individual Conv2D. In Each block, 3 Conv2D layers are there. Model-2 has 3 such a Conv2D blocks are there and each block follows Maxpooling2D at the end. Model-2 has three FC layers among that first two having 512 nodes and last having 2 nodes. In the last FC network, we have organizeda sigmoid activation function that will classify and give probabilities of input being in a nodule or non-nodule class. Model-2 is very similar to LcdNet proposed by Giang Son Tran el [11]. To select the best architecture among these two we have performed some of the operations that will optimize our architectures and give the best performance.

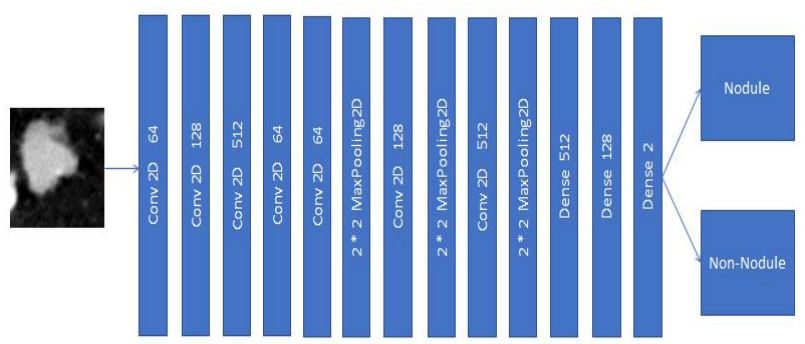

Figure 3: Model - 1(Lung Nodule Detection Net (LndNet))

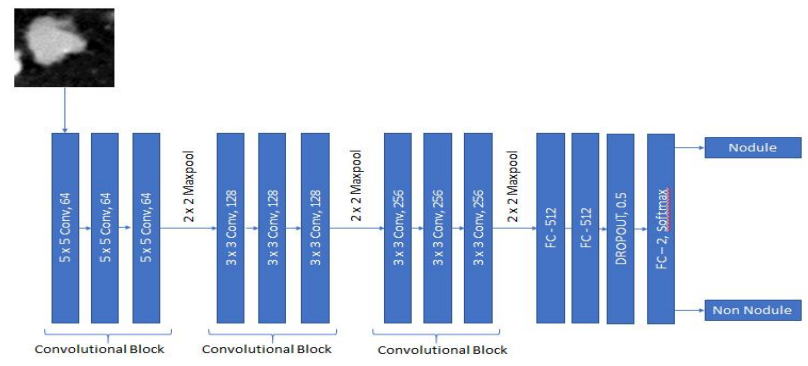

Figure 4: Model-2 (2DCNN block architecture)

\subsection{Activation functions}

We have trained both of the architectures with three well known activation functions ReLU, LeakyReLU and ELU [21] [table 6]. We have measured the performance concerning 
Accuracy, Sensitivity, and Specificity. Our overall objective is to find out a more sensitive network that can produce high sensitivity because our projector system is a nodule-non-nodule detection system in lung CT scans. Cancer detection networks must be sensitive. The below table $\mathrm{V}$ and graph suggested that Model-1 with ReLU Activation Function gives High performance in three of the aspects. LeakyReLU equally performs well. For the initial stage, we have stared with binary crossentropy loss function and adam as an optimizer.

Table 5: Model-1 and Model-2 using different activation functions

\begin{tabular}{|l|l|l|l|l|}
\hline Model & Activation & Specificity & Accuracy & Sensitivity \\
\hline M-1 & ELU & 97.16 & 94.01 & 81.2 \\
\hline M-2 & ELU & 96.56 & 92.97 & 73.04 \\
\hline M-1 & LeakyReLU & $\mathbf{9 6 . 8 2}$ & $\mathbf{9 4 . 6 4}$ & $\mathbf{8 6}$ \\
\hline M-2 & LeakyReLU & 92.68 & 91.49 & 85.81 \\
\hline M-1 & ReLU & 96.34 & 94.45 & 86 \\
\hline M-2 & ReLU & 91.27 & 90.56 & 87.23 \\
\hline
\end{tabular}

Table 6: ELU, LeakyRelu and Relu activation functions comparisons

\begin{tabular}{|c|c|c|c|}
\hline \multicolumn{4}{|c|}{ [24] } \\
\hline $\begin{array}{l}\text { Sr. } \\
\text { No }\end{array}$ & Activation Functions & Advantages & Disadvantages \\
\hline 1. & $\begin{array}{l}\text { ELU } \\
R(z) \\
=\left\{\begin{array}{cc}z, & z>0 \\
\alpha .\left(e^{2}-1\right) & z \leq 0\end{array}\right.\end{array}$ & $\begin{array}{l}\text { It can pro- } \\
\text { duce nega- } \\
\text { tive outputs, } \\
\text { becomes a } \\
\text { smooth and } \\
\text { slow and } \\
\text { good alter- } \\
\text { native for } \\
\text { ReLU. }\end{array}$ & $\begin{array}{l}\text { For } z>0 \text {, it can } \\
\text { blow up the } \\
\text { activation out- } \\
\text { put range of } \\
{[0, \infty] .}\end{array}$ \\
\hline 2. & $\begin{array}{l}\text { ReLU } \\
\qquad R(z)=\left\{\begin{array}{l}z, z>0 \\
0, z \leq 0\end{array}\right.\end{array}$ & $\begin{array}{l}\text { It can re- } \\
\text { solve va- } \\
\text { nishing gra- } \\
\text { dient prob- } \\
\text { lem. It is } \\
\text { less compu- } \\
\text { tationally } \\
\text { expensive } \\
\text { than other } \\
\text { activation } \\
\text { functions. }\end{array}$ & $\begin{array}{l}\text { We can use it } \\
\text { within the hid- } \\
\text { den layers only. } \\
\text { It may lead to a } \\
\text { vanishing gra- } \\
\text { dient problem. } \\
\text { It will blow up } \\
\text { the activation } \\
\text { because of its } \\
\text { range. }\end{array}$ \\
\hline 3. & $\begin{array}{l}\text { LeakyReLU } \\
\quad R(z) \\
\quad=\left\{\begin{array}{rr}z, & z>0 \\
\alpha . z, & z \leq 0\end{array}\right.\end{array}$ & $\begin{array}{l}\text { We can use } \\
\text { it to fix the } \\
\text { dying ReLU } \\
\text { problem by } \\
\text { putting some } \\
\text { negative } \\
\text { slop. }\end{array}$ & $\begin{array}{l}\text { We cannot use } \\
\text { complex classi- } \\
\text { fication prob- } \\
\text { lems. } \\
\text { In some of the } \\
\text { cases, it per- } \\
\text { forms bad than } \\
\text { Tanh and Sig- } \\
\text { moid. }\end{array}$ \\
\hline
\end{tabular}

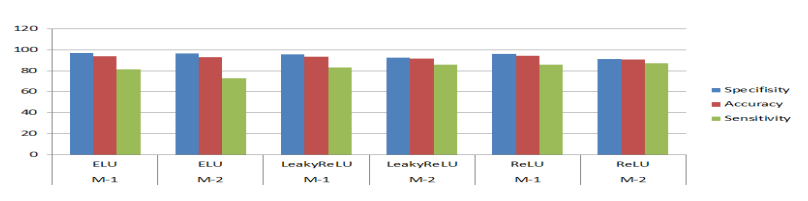

Figure 5: Graph of Above Table 5.

\subsection{Loss Functions}

At the initial stage for identifying best architecture we have tested three loss functions like Binary-cross entropy (BCE),
Hinge and Squared Hinge loss on two models with LeakyReLU Activation function. Based on the experiment and according to the functionality of the loss functions and for our binary classification problem best loss function is BCE. We have shown an experimental result graph that justifies BCE is preforming well among all three loss functions.

Table 7: Loss Functions Experimental Results Using LeakyreluActivation Function.

\begin{tabular}{|c|c|c|c|c|}
\hline \multirow{3}{*}{ Model } & $\begin{array}{l}\text { Loss } \\
\text { Function }\end{array}$ & Specificity & Accuracy & Sensitivity \\
\hline \multirow{3}{*}{ M-1 } & BCE & $\mathbf{9 6 . 3 4}$ & $\mathbf{9 4 . 4 5}$ & $\mathbf{8 7}$ \\
\cline { 2 - 5 } & Hinge & 96.04 & 93.77 & 82 \\
\cline { 2 - 5 } & $\begin{array}{c}\text { Squared- } \\
\text { Hinge }\end{array}$ & 97.4 & 94.08 & 78.4 \\
\hline \multirow{3}{*}{ M-2 } & BCE & 92.68 & 91.49 & 85.81 \\
\cline { 2 - 5 } & Hinge & 96.56 & 92.47 & 73.05 \\
\cline { 2 - 5 } & $\begin{array}{c}\text { Squared- } \\
\text { Hinge }\end{array}$ & 94.4 & 85.69 & 50.26 \\
\hline
\end{tabular}

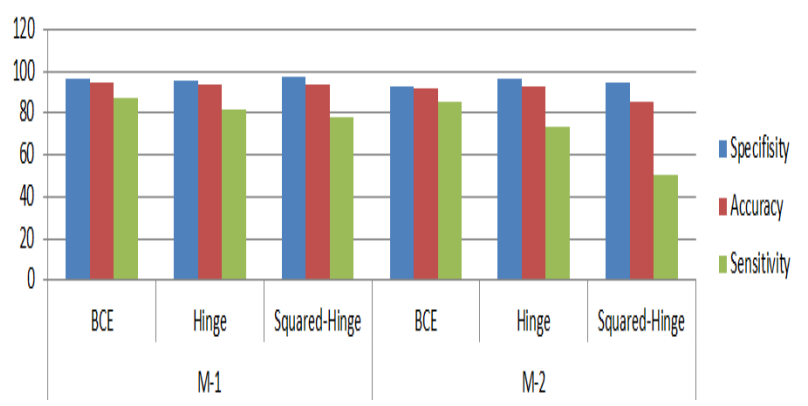

Figure6: Graph of Above Table 7.

\subsection{Optimizers}

For now, we have finalizedRelu/LeakyReLU as an activation function and BCE as a loss function for M-1 and M-2. Now with this background our next experiment for three different optimizers like Adam, Rmsprop and Stochastic gradient (SGD). The below table VIII shows experimental results on M-1, M-2 with activation functions ReLU/Leakey ReLU and optimizer BCE. After all these experiments we have finalized our CNN architecture with 7 convolution2D layers, 3 MaxPooling2D layer, and 3 fully-connected layers. For more optimization of the model, we have also tested for the best batch size and number of Convolution layers. We have tested 6,7,8,9 convolution layer networks with different batch sizes like 32, 64,128 and 256. Among all 7 layer convolution networks with 128 batch sizes have the best accuracy, sensitivity, and specificity. Finally, we have optimized our architecture with the LUNA16 dataset which is currently the smallest architecture for the classification of nodule and non-nodule candidates. For the sake of inquisitiveness, we have also tried LeakeyReLUactivation function with adam optimizer and that combination gives us high Accuracy, Sensitivity, and Specificity with compare to all other combinations. Table VIII shown below shows the results for the same. 
Table 8: Experiments with different optimizers

\begin{tabular}{|l|l|l|l|l|}
\hline Model & Optimizer & Accuracy & Sensitivity & Specificity \\
\hline M-1 & Adam with ReLU & 95 & 87 & 97.3 \\
\hline M-1 & $\begin{array}{l}\text { Adam with Lea- } \\
\text { kyReLU }\end{array}$ & $\mathbf{9 5 . 2}$ & $\mathbf{9 0 . 1}$ & $\mathbf{9 7 . 2}$ \\
\hline M-1 & RMSProp & 94.14 & 85.10 & 96.04 \\
\hline M-1 & SGD & 94.63 & 80.14 & 97.68 \\
\hline M-2 & Adam & 90.56 & 85.5 & 91.26 \\
\hline M-2 & RMSProp & 90.57 & 85.6 & 91.27 \\
\hline M-2 & SGD & 94.38 & 77.66 & 97.91 \\
\hline
\end{tabular}

Till now we have optimized our architecture and got reasonable accuracy compared to other researchers. Our aim to make such a CAD system that can classify nodule or non-nodule. Cancer detection systems must be sensitive enough that can identify cancer even at a very early stage. So after this stage, our focus is to make architecture or model sensitive that can decrease the false-negative ratio. As a result, we can reduce the chances of a patient having cancer but not detected by the system.

\subsection{Making our model Sensitive}

To do so we thought will training if we give more weightage to the minority class in our case CT scan containing nodule. Data is so imbalanced in our case. Data augmentation was not enough to increase accuracy and sensitivity. So we decided that make such a loss function that can give more weightage to minority class and less weightage to the majority class while training according to their frequency. By this thought, we decided to modify binary loss function in such a way that it will become more sensitive towards minority class. Binary cross-entropy function defied as $\mathrm{y}=-\mathrm{y} \log (\mathrm{p})-(1-\mathrm{y}) \log (1-\mathrm{p})$, where $\mathrm{y}$ is the class binary indicator ( 0 or 1$)$ and $\mathrm{p}$ is predicted probability, for instance, belonging to class 1 . To incorporate the weights of two classes ( 0 and 1$)$ into the cross-entropy, one can define sensitive cost function: $\mathrm{y}=-\mathrm{w} \_\mathrm{ylog}(\mathrm{p})-\mathrm{w} \_1(1-\mathrm{y}) \log (1-\mathrm{p})$, in which $\mathrm{w}_{-} 0$ and $\mathrm{w}_{-} 1$ are the weights for class 1 and 0 , respectively.

\subsection{Mobile application development using TensorFlow lite}

To provide our CAD system real-time access and mobilityit is better to transfer this all things into the mobile application so it can add more dimension to it. For transferring this thing into android mobile application we have used the TensorFlow lite framework.

Algorithm for Lung nodule detection mobile application:

1. Save your trained model.h5 or model.hdf5 (This file will have optimized weights)

2. Transfer this model.h5 in to TensorFlow lite model (file extension is .tflite)

3. Save your trained model.h5 or model.hdf5 (This file will have optimized weights)

4. Transfer this model.h5 in to TensorFlow lite model (file extension is .tflite )

5. If your model size still very large after converting into .tflite you can convert .tflite file into .pb file. It will occupy less size of your model

6. Make appropriate GUI for android application [Fig. 7] Load your .pb or .tflite file in your application

7. Load some testing files and test with application. [Fig. 7]

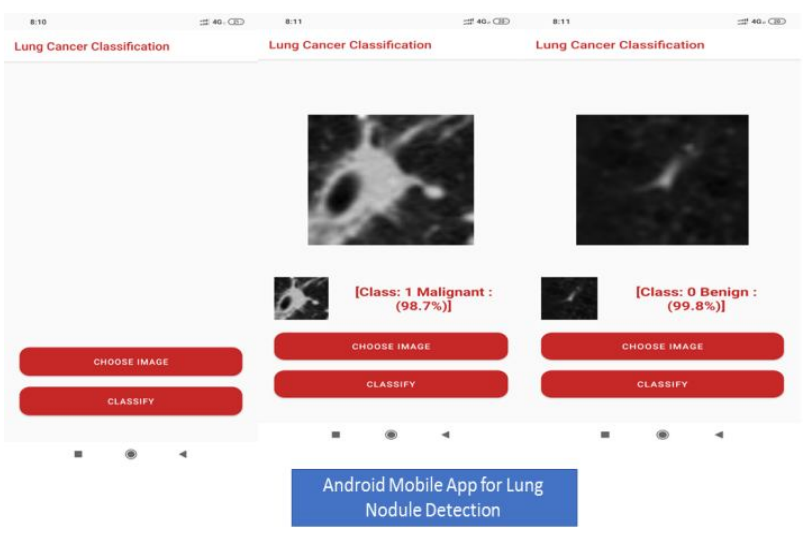

Figure 7: GUI for our LndNet android application

\section{EXPERIMENTAL RESULTS}

\subsection{Experimental setup}

A. Preprocessing:

For the standardization of CT scan images, we required to accomplish data preprocessing. Firstly we have converted each 3D CT scan slice into a 2D grayscale .jpeg image. We abstract one non-overlying image spot of size pixels for each annotation of both nodule and non-nodule examples. We have removed observations that are very near to the boundaries of slices $(<40$ pixels). Figure 8 illustrates some extracted image patches using this method. The extracted image's intensity I am then scaled to the Hounsfield unit $\left.\mathrm{I}_{\mathrm{HU}} \in[-1000,400]\right)$ and linearly normalized to range using the transformation $I_{n o r m}=\left(I_{H U}+1000\right) / 1400$ before being used as input for training the model (figure 8).

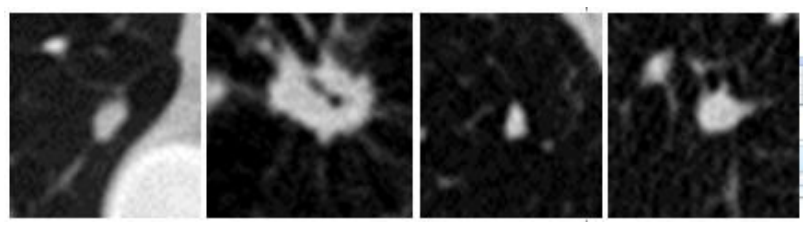

Figure 8:Some of the extracted ROI from LUNA16 data

B. Performance measurement:

Accuracy (capability to distinguish benign and malignant nodule cases correctly), sensitivity (capability to define the malignant nodule cases correctly), and specificity (capability to define the benign nodule cases correctly) are used to quantify the exactness of the classification. Generally, for binary classification problems, these kinds of metrics are widely used and equations for them as follow:

$$
\begin{gathered}
\text { ACCURACY }=\frac{T P+T N}{T P+T N+F P+F N} \\
\text { SENSITIVITY }=\frac{T P}{T P+F N} \\
\text { SPECIFICITY }=\frac{T N}{T N+F P}
\end{gathered}
$$

Where, TP: True Positives, TN: True Negatives, FP: False Positives, FN: False Negatives.

C.

D. Systems configuration:

Hardware: We have implemented our experimental results on Intel(R) Core(TM) i-7-8700k CPU with 3.70GHz, 32 GB RAM 
and 64 bit-operating system. This has support of NVIDIA graphics card. The configuration of the Graphics card is NVIDIA GFORCE TITAN Xp with 3840 Cuda cores. Some of the experiments we have done on Kaggle cloud platform they are providing tesla K10 GPU for experimental purpose.

Software: We implement our system using Keras 2.1.1 with TensorFlow 1.3 as the backend, along with CUDA 9.0 for GPU acceleration. Our CAD system is implemented with Python 3.6.5.

\section{E. Outcomes and Deliberations}

For the lung nodule detection system, we have followed two different approaches to boost up our classifier's accuracy.

Table 9:LndNet and other pre-train model testing results

\begin{tabular}{|l|l|l|l|l|l|l|}
\hline $\begin{array}{l}\text { Sr. } \\
\text { No }\end{array}$ & Models & Dataset & $\begin{array}{l}\text { Sca } \\
\text { ns }\end{array}$ & $\begin{array}{l}\text { Accu- } \\
\text { racy }\end{array}$ & $\begin{array}{l}\text { Sensi- } \\
\text { tivity }\end{array}$ & $\begin{array}{l}\text { Speci- } \\
\text { ficity }\end{array}$ \\
\hline $\mathbf{1}$ & $\begin{array}{l}\text { LndNet } \\
\text {-BCE }\end{array}$ & $\begin{array}{l}\text { LIDC/I } \\
\text { DRI }\end{array}$ & $\mathbf{8 8 8}$ & $\mathbf{9 5 . 2 5}$ & $\mathbf{8 9 . 2 3}$ & $\mathbf{9 7 . 2}$ \\
\hline 2 & $\begin{array}{l}\text { Res- } \\
\text { Net50 }\end{array}$ & $\begin{array}{l}\text { LIDC/I } \\
\text { DRI }\end{array}$ & 888 & 94.14 & 89.007 & 94.02 \\
\hline 3 & $\begin{array}{l}\text { Goog- } \\
\text { leNet or } \\
\text { Incep- } \\
\text { tionV3 }\end{array}$ & $\begin{array}{l}\text { LIDC/I } \\
\text { DRI }\end{array}$ & 888 & 93.77 & 81.97 & 96.26 \\
\hline 4 & $\begin{array}{l}\text { VGGNe } \\
\text { t(16) }\end{array}$ & $\begin{array}{l}\text { LIDC/I } \\
\text { DRI }\end{array}$ & 888 & 93.46 & 82.59 & 95.63 \\
\hline 5 & $\begin{array}{l}\text { AlexNet } \\
\text { LIDC/I } \\
\text { DRI }\end{array}$ & 888 & 93.52 & 89.007 & 94.47 \\
\hline 6 & LeNet & $\begin{array}{l}\text { LIDC/I } \\
\text { DRI }\end{array}$ & 888 & 88.47 & 72.94 & 90.74 \\
\hline
\end{tabular}

Approach 1: Using sensitive cost loss function

By giving different values of sensitive cost function variables w0 and w1 we have increased our classifier's performance. The below table $\mathrm{X}$ shows experimental results of ourNetworkLcdNetCost-sensitive function (CSF) having different batch sizes and epoch values. These experiments we have done without the k-folding cross-validation technique. After applying stratified k-folding techniques we have achieved more accurate results showed intable 11 .

Table 10: Sensitive Cost Function Experiments

\begin{tabular}{|c|c|c|c|c|c|c|c|}
\hline \multirow[b]{2}{*}{$\begin{array}{l}\text { Sr. } \\
\text { No. }\end{array}$} & \multicolumn{2}{|c|}{$\begin{array}{l}\text { Input Net- } \\
\text { work }\end{array}$} & \multicolumn{2}{|c|}{$\begin{array}{c}\text { Sensitive cost } \\
\text { function }\end{array}$} & \multicolumn{3}{|c|}{ Performance } \\
\hline & $\begin{array}{l}\text { Epo } \\
\text { chs }\end{array}$ & $\begin{array}{l}\text { Batch } \\
\text { Size } \\
\end{array}$ & W0 & W1 & $\begin{array}{l}\text { Ac- } \\
\text { cura- } \\
\text { cy }\end{array}$ & $\begin{array}{l}\text { Sensi- } \\
\text { tivity }\end{array}$ & $\begin{array}{l}\text { Speci- } \\
\text { ficity }\end{array}$ \\
\hline 1 & 20 & 32 & 0.1 & 0.9 & 91.1 & 78.3 & 95.5 \\
\hline 2 & 30 & 32 & 0.2 & 0.8 & 93.56 & 82.3 & 96.3 \\
\hline 3 & 50 & 32 & 0.3 & 0.7 & 92.9 & 84.2 & 96.7 \\
\hline 4 & 25 & 32 & 0.2 & 0.8 & 93.2 & 84.5 & 97.43 \\
\hline 5 & 20 & 64 & 0.4 & 0.5 & 94.53 & 86.3 & 97.22 \\
\hline 6 & 30 & 64 & 0.5 & 0.5 & 93.2 & 88 & 97.23 \\
\hline 7 & 25 & 64 & 0.3 & 0.7 & 95 & 79.85 & 97.1 \\
\hline 8 & 25 & 128 & 0.6 & 0.4 & 96 & 95.7 & 97.3 \\
\hline 9 & 25 & 128 & 0.5 & 0.5 & 95.2 & 93.2 & 96.1 \\
\hline 10 & 25 & 256 & 0.5 & 0.5 & 94.83 & 90.1 & 97 \\
\hline
\end{tabular}

We have tested our network with $\mathrm{k}$-folding validation testing. In which stratified $k$-folding $(\mathrm{k}=10)$ has given high accuracy. Our LndNet with CSFand stratified k-folding has produced promising results and these results are very much similar to LcdNetFL-Giang Son Tran and LcdNet-CE Giang Son Tran[20] work. Accuracy and specificity little less than the work suggested by Giang Son but we can able to reach high sensitivity with our LndNet-CSF in table 9. we have provided a comparison table 11 of related work results on the LUNA16 dataset in terms of accuracy, sensitivity, and specificity.

Table 11:SCF and validation techniques results

\begin{tabular}{|c|c|c|c|c|c|}
\hline $\begin{array}{l}\text { Sr } \\
\text { No }\end{array}$ & $\begin{array}{l}\text { Sen- } \\
\text { sitive } \\
\text { cost } \\
\text { func- } \\
\text { tion }\end{array}$ & $\begin{array}{l}\text { Valida- } \\
\text { tion } \\
\text { technique }\end{array}$ & $\begin{array}{l}\text { Accu- } \\
\text { racy }\end{array}$ & $\begin{array}{l}\text { Sensi- } \\
\text { tivity }\end{array}$ & $\begin{array}{l}\text { Speci- } \\
\text { ficity }\end{array}$ \\
\hline 1 & No & $\begin{array}{l}\text { Without } \\
\text { k-folding }\end{array}$ & 96 & 95.7 & 97.3 \\
\hline 2 & Yes & k-folding & 96.1 & 95.9 & 97.5 \\
\hline 3 & Yes & $\begin{array}{l}\text { With } \\
\text { stratified } \\
\text { k-folding }\end{array}$ & $\mathbf{9 6 . 9}$ & $\mathbf{9 6 . 2}$ & $\mathbf{9 7 . 2}$ \\
\hline
\end{tabular}

\section{Approach 2: Progressive resizing or scaling}

Progressive resizing is a technique for building CNNs that can be very helpful during the training and optimization phases of a machine learning project. Here we have given different steps that we have performed while applying Progressive resizing or scaling to our LUNA16 CT scan dataset.

1. Download and format the data.

2. Define the data generators: Data generators are on the fly image transformers and are the recommended way of providing image data to models in Keras. They let you work with on-disk image data too large to fit all at once inmemory. And they allow you to preprocess the images your model sees withrandom image transformations and standardizations, a key technique for improving model performance.

3. Define the model.

4. Fit the model.

5. Applying progressive resizing: To build a classifier we have started to form tiny $\mathrm{n} \times \mathrm{n}(20 \times 20)$ imagesthat perform well. The second step is resizing our model up to $2 n \times 2 n(40 \times 40)$ images. We do this using transfer learning. Transfer learning is the technique of reusing layers and weights from previous models when building new ones.

6. Define the model.

7. Fit the model.

8. Applying progressive resizing: To build a classifier we have started to form tiny $\mathrm{n} \times \mathrm{n}(20 \times 20)$ imagesthat perform well. The second step is resizing our model up to $2 \mathrm{n} \times 2 \mathrm{n}(40 \times 40)$ images. We do this using transfer learning. Transfer learning is the technique of re-using layers and weights from previous models when building new ones. 
Table 12: Comparison table with other works

\begin{tabular}{|c|c|c|c|c|c|}
\hline \multirow[b]{2}{*}{ Work } & \multirow[b]{2}{*}{$\begin{array}{c}\text { scan } \\
\text { S }\end{array}$} & \multirow[b]{2}{*}{$\begin{array}{c}\text { Data- } \\
\text { set }\end{array}$} & \multicolumn{3}{|c|}{ Performance } \\
\hline & & & $\begin{array}{c}\text { Ac- } \\
\text { cura- } \\
\text { cy }\end{array}$ & $\begin{array}{l}\text { Sensi- } \\
\text { tivity }\end{array}$ & $\begin{array}{l}\text { Speci- } \\
\text { ficity }\end{array}$ \\
\hline $\begin{array}{l}\text { Pro- } \\
\text { posed } \\
\text { LndNet- } \\
\text { CSF }\end{array}$ & 888 & $\begin{array}{c}\text { LIDC/ } \\
\text { IDRI }\end{array}$ & 96.9 & 96.2 & 97.2 \\
\hline $\begin{array}{l}\text { LcdNet- } \\
\text { FL-Giang } \\
\text { Son } \\
\text { Tran[11] }\end{array}$ & 888 & $\begin{array}{c}\text { LIDC/I } \\
\text { DRI }\end{array}$ & 97.2 & 96 & 97.3 \\
\hline $\begin{array}{l}\text { LcdNet- } \\
\text { CE- } \\
\text { Giang } \\
\text { Son } \\
\text { Tran[11] }\end{array}$ & 888 & $\begin{array}{c}\text { LIDC/I } \\
\text { DRI }\end{array}$ & 95.6 & 90.2 & 96 \\
\hline Setio[15] & 888 & $\begin{array}{c}\text { LIDC/I } \\
\text { DRI }\end{array}$ & 95 & 91.1 & - \\
\hline $\begin{array}{l}\text { Li et al. } \\
{[12]}\end{array}$ & 1010 & $\begin{array}{c}\text { LIDC/I } \\
\text { DRI }\end{array}$ & 86.4 & 87.1 & \\
\hline $\begin{array}{l}\text { Kuruvilla } \\
\text { and Gu- } \\
\text { navathi } \\
{[13]}\end{array}$ & 155 & $\begin{array}{c}\text { LIDC/I } \\
\text { DRI }\end{array}$ & 93.3 & 91.4 & 100 \\
\hline $\begin{array}{l}\text { Choi and } \\
\text { Choi [14] }\end{array}$ & 58 & $\begin{array}{c}\text { LIDC/I } \\
\text { DRI }\end{array}$ & 97.6 & 95.2 & 96.2 \\
\hline
\end{tabular}

9. Appends a max-pooling layer, which downsamples $40 \times 40 \rightarrow 20 \times 20$ - the expected input size for our old model.

10. Removes the first convolutional layer from our old model (it helps in reducing overfitting).

11. Reattaches rest of the layers of our old model into the new one.

12. Freeze old convolutional and fully-connected layer weights in place.

13. Repeat steps 5 to 12 to create a new model for $80 \mathrm{x}$ 80 image size.

Below table showing experimental results of progressive sizing with transfer learning

Table 13: Progressive resizing results on luna16 dataset

\begin{tabular}{|c|c|c|c|c|}
\hline Sr.No: & $\begin{array}{c}\text { Image } \\
\text { size }\end{array}$ & Accuracy & Sensitivity & Specificity \\
\hline 1 & $20 \times 20$ & 90.23 & 78.38 & 96.0 \\
\hline 2 & $40 \times 40$ & 94.20 & 85.16 & 96.5 \\
\hline 3 & $80 \times 80$ & 96.2 & 91.2 & 97.8 \\
\hline
\end{tabular}

\section{CONCLUSION AND FUTURE WORK}

In Lung nodule detection application sensitivity of a network is a very important part. To improve the model accuracy and sensitivity our proposed LndNet-SCF and progressive resizing approaches have produced very promising results. For further enhancement, one can try with 3D images and gather a history of the patient that maybe help full for getting more accuracy. For more sensitivity, one can increase w0 value in CSF but more value may decrease the accuracy and specificity of the classification result. Using Deep CNN with CSF is an excellent classifier with $96.9 \%$ accuracy, $96.2 \%$ sensitivity, and $97.2 \%$ specificity.

\section{REFERENCES}

1. Lung cancer is responsible for $\mathbf{1}$ in $\mathbf{5}$ cancer deaths worldwide and largely preventable, Available online: The cancer atlas http://canceratlas.cancer.org/(accessed on 1 July 2019).

2. Centers for Disease Control and Prevention, Lungcancer statistics. https://www.cdc.gov/cancer/lung/( accessed on 1 August 2019).

3. K. Doi. Computer-aided diagnosis in medical imaging: historical review, current status and future potential, Computerized Medical Imaging and Graphics, vol. 31, no. 4-5, pp. 198-211, 2007.

4. L. A. Torre, R. L. Siegel, and A. Jemal, Lung cancer statistics, in Lung Cancer and Personalized Medicine,Springer, pp. 1-19, Berlin, Germany, 2016. https://doi.org/10.1007/978-3-319-24223-1_1

5. Q. Song, L. Zhao, X. Luo, and X. Dou, Using deep learning for classification of lung nodules on computed tomography images, Journal of Healthcare Engineering, vol. 2017, Article ID 8314740, 7 pages, 2017. https://doi.org/10.1155/2017/8314740

6. T.-Y. Lin, P. Goyal, R. Girshick, K. He, and P. Dollár,Focal loss for dense object detection, in Proceedings of 2017 IEEE International Conference on Computer Vision (ICCV), Venice, Italy,pp. 2999-3007.

7. Y. Lecun, L. Bottou, Y. Bengio, and P. Haffner. Gradient-based learning applied to document recognition, Proceedings of the IEEE 1998, vol. 86, no. 11, pp. 2278-2324.

8. A. Krizhevsky, I. Sutskever, and G. E. Hinton. ImageNet classification with deep convolutional neural networks, in Proceedings of the 25th International Conference on Neural Information Processing Systems, Curran Associates Inc., Lake Tahoe, NV, USA, December 2012. Volume 1, NIPS'12, pp. 1097-1105.

9. K. Simonyan and A. Zisserman.Very deep convolutional networks for large-scale image recognition, in Proceedings of IEEE International Conference on Learning Representations, Banff, AB, Canada, April 2014.

10. K. He, X. Zhang, S. Ren, and J. Sun, Deep residual learning for image recognition, in Proceedings of 2016 IEEE Conference on Computer Vision and Pattern Recognition (CVPR), Las Vegas, NV, USA, June 2016, pp. 770-778.

11. Giang Son Tran, Thi Phuong Nghiem. Improving Accuracy of Lung Nodule Classification Using DeepLearning with Focal Loss, Journal of Healthcare Engineering, Article ID 5156416, https://doi.org/10.1155/2019/5156416, Volume 2019.

12. W. Li, P. Cao, D. Zhao, and J. Wang. Pulmonary nodule classification with deep convolutional neuralnetworks on computed tomography images,Computational and Mathematical Methods in Medicine, vol. 2016, Article ID 6215085, 7 pages, 2016.

13. J. Kuruvilla and K. Gunavathi, Lung cancer classification using neural networks for CT images,Computer Methods and Programs in Biomedicine, vol. 113, no. 1, pp. 202-209, 2014.

14. W.-J. Choi and T.-S. Choi. Automated pulmonary nodule detection system in computed tomography 
images: a hierarchical block classification approach, Entropy, vol. 15, no. 2, pp. 507-523, 2013.

15. A. A. A. Setio, F. Ciompi, G. Litjens et al. Pulmonary nodule detection in $\mathrm{CT}$ images: false positive reduction using multi-view convolutional net-works, IEEE Transactions on Medical Imaging, vol. 35, no. 5, pp. 1160-1169, 2018.

16. E. L. Torres, E. Fiorina, F. Pennazio et al. Large scale validation of the M5L lung CAD on heterogeneous CT datasets, Medical Physics, vol. 42, no. 4, pp. 14771489, 2015.

17. A. A. A. Setio, A. Traverso, T. de Bel et al. Validation, comparison, and combination of algorithms for automatic detection of pulmonary nodules in computed tomography images: the LUNA16 challenge, Medical Image Analysis, vol. 42, pp. 1-13, 2017.

18. S. G. Armato III, G. McLennan, L. Bidaut et al. The lung image database consortium (LIDC) and image database resource initiative (IDRI): a completed reference database of lung nodules on ct scans, Medical Physics, vol. 38, no. 2, pp.915-931, 2011.

19. CNN Architectures: LeNet, AlexNet, VGG, GoogLeNet, ResNet and more. Online available: Analytics Vidhya, https://medium.com/analytics-vidhya/cnnsarchitectures-lenet-alexnet-vgg-googlenet-resnet-andmore-666091488df5(accessed on 1 July 2018).

20. Kaggle. Lung Nodule Analysis 2016, LUN16 competition data.

21. H. D. Gadadeand Dr. D.K.Kirange. Machine Learning Approach towards Tomato Leaf Disease Classification, International Journal of Advanced Trends in Computer Science and Engineering, Volume 9, No.1, pp. 490-495, January - February 2020 https://doi.org/10.30534/ijatcse/2020/67912020

22. Ali Mohammad Alqudah, HiamAlquraan, Isam Abu Qasmieh, Amin Alqudah and Wafaa Al-Sharu. Brain Tumor Classification Using Deep Learning Technique - A Comparison between Cropped, Uncropped, and Segmented Lesion Images with Different Sizes, Volume 8, No.6, pp. 3684-3691, NovemberDecember 2019.

https://doi.org/10.30534/ijatcse/2019/155862019

23. Edward B. Panganiban, Arnold C. Paglinawan, Wen Yaw Chung, and Gilbert Lance S. Paa. A Novel Technique in Classifying Heart Diseases based on Electrocardiogram (ECG) Signals using Deep Learning and Spectrogram Image Analysis, Volume 8, No.4, pp. 3684-3691, July-August 2019. https://doi.org/10.30534/ijatcse/2019/102842019

24. Keras Documentation, Available Online: Activations, https://keras.io/activations/(accessed on 1 January 2020). 